\title{
Type of Valve Dysfunction
}

National Cancer Institute

\section{Source}

National Cancer Institute. Type of Valve Dysfunction. NCI Thesaurus. Code C90323.

A finding that indicates the specific type of abnormality that is present in a heart valve such as insufficiency, regurgitation or prolapse. 